\title{
A Passive, Real-time, Terahertz Camera for Security Screening, using Superconducting Microbolometers
}

\author{
E.N. Grossman, C.R. Dietlein, M. Leivo, A. Rautiainen, and A. Luukanen ${ }^{2}$ \\ ${ }^{1}$ Optoelectronics Division, National Institute of Standards and Technology, Boulder, CO 80304, USA \\ ${ }^{2}$ VTT Technical Research Center of Finland, Espoo, Finland
}

\begin{abstract}
We describe a broadband terahertz camera based on a modular 64-element linear array of hot-spot microbolometers Unlike many superconducting sensor arrays, the readout for this array is performed by entirely uncooled electronics; no SQUIDs or cryogenic HEMTs are employed. The operating principles for the microbolometer and the readout scheme are described and compared with those of similar superconducting sensors.
\end{abstract}

Index Terms - Microbolometer, superconducting, terahertz, camera

\section{INTRODUCTION}

Terahertz (0.1-1 THz) imaging has been used for decades, mainly in radio astronomy. However, it is presently of intense interest for security screening of personnel[1], which requires a completely new level of user-friendliness, reliability, and robustness. The capability that $\mathrm{THz}$ frequency offers is a combination of good penetration through obscurants (clothing in particular) with good diffraction-limited spatial resolution. Other things being equal, passive imaging is preferred over actively illuminating the target, partly because of public acceptance issues with irradiating people, and partly because of the limited capabilities and high cost of $\mathrm{THz}$ sources. However, the sensitivity levels required for useful passive screening of people are highly challenging, particularly indoors, where background temperature contrasts are low. Superconducting sensors offer a realistic means of attaining this sensitivity. They can be incorporated into the necessary array sizes much more straightforwardly than uncooled coherent (heterodyne) sensors, which are the only realistic uncooled alternative.

Fig. 1 illustrates this sensitivity challenge. Passive radiometer sensitivity is ordinarily specified in terms of noiseequivalent temperature difference (NETD), the minimum contrast in brightness temperature that can be detected with unity signal-to-noise ration (SNR) in a specified integration time, conventionally $1 / 30 \mathrm{~s}$. The radiation being imaged is simply the Rayleigh-Jeans tail of the blackbody emission from the target, along with reflected blackbody emission from the background. Indoors, the resulting contrasts are therefore generally $<20 \mathrm{~K}$. The images in Fig. 1 are absolutely calibrated in terms of brightness temperature (a calibrator lies to the right of the subject's head). Each contains the same broadband passive image of a seated male subject concealing a ceramic knife beneath a synthetic fleece. The original image was taken with a single-pixel superconducting sensor, with an NETD of $\sim 200 \mathrm{mK}$. Varying amounts of Gaussianlydistributed random noise were then added in each different panel in order to simulate the same scene, as observed with sensors of progressively poorer sensitivity.
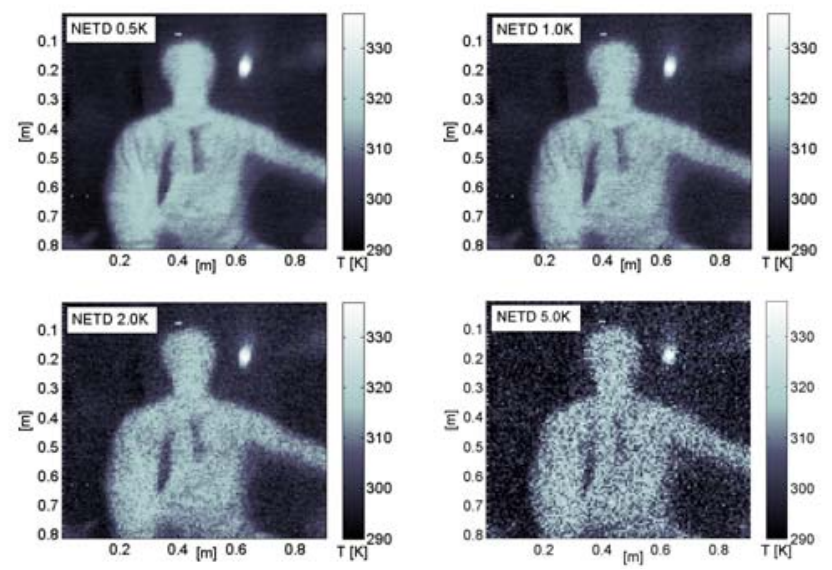

Fig. 1 Images of a typical $\mathrm{THz}$ concealed weapons detection scenario, with noise added to simulate observation by sensors of $\mathrm{NETD}=0.5 \mathrm{~K}, 1 \mathrm{~K}, 2 \mathrm{~K}$, and $5 \mathrm{~K}$.

This sequence of images illustrates several points regarding the application requirements. First, threat items can have signal strengths up to $5-10 \mathrm{~K}$. Second, realistic $\mathrm{THz}$ scenes contain substantial clutter however, both in the background and on the target itself, at levels up to $1-2 \mathrm{~K}$ (an example is the folds of the clothing,) that could easily prevent detection of weaker threat signatures. Third, the eye can distinguish and reject this clutter when the sensitivity and spatial resolution of this image are adequate. Thus, the required NETD for this type of screening is approximately $0.5 \mathrm{~K}$. This corresponds, in a $100 \mathrm{GHz}$ bandwidth, to an NEP of 0.7 $\mathrm{pW} / \mathrm{Hz}^{1 / 2}$, well beyond the present state-of-the-art for incoherent, uncooled $\mathrm{THz}$ sensors.

\section{SENSOR Design, PRinciPle OF OpERATION, AND PERFORMANCE}

The heart of the camera is a linear 1x64 array of superconducting microbolometers. The array is organized into 8 -element modules, each containing a $5 \times 24 \mathrm{~mm}$ device die in an overall $25 \times 25 \mathrm{~mm}$ package layout (See Fig. 2). The modularity is important (a) to mitigate the effects of 


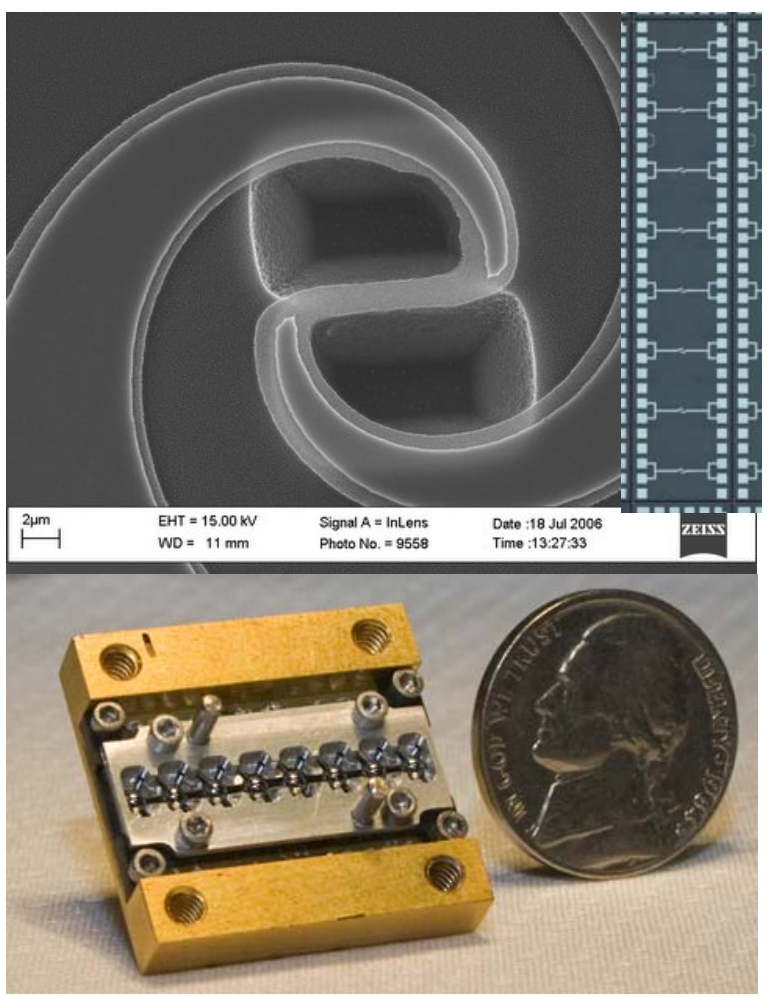

Fig. 2 Closeup of an individual microbolometer, with 8element die in the inset. (Below) a packaged 8-element module showing the hyperhemispherical substrate lenses.

imperfect fabrication yield and detector uniformity, (b) to enable easy reconfiguability (e.g. $1 \times 64->2 \times 32$ ) of the focal plane, and (c) to enable future curved focal planes, which can greatly ease the optical design of very wide-field systems.

The detectors are antenna-coupled, superconducting hotspot microbolometers, cooled to $\sim 4 \mathrm{~K}$, whose physics and performance have been described elsewhere[2, 3]. NETD levels of $115 \mathrm{mK}$ have been reached. They consist of simple strips of $\mathrm{Nb}$ or $\mathrm{NbN}$ thin films placed between the feedpoints of a lithographic antenna, and micromachined so as to form a free-standing bridge, suspended above the substrate (see Fig. 2). As the name implies, a hot-spot forms at the center of the bridge in operation, where the temperature exceeds the superconducting transition temperature $\left(\mathrm{T}_{\mathrm{c}}\right)$ and the metal is resistive. As the $\mathrm{THz}$ power dissipated in the bridge varies, so varies the length of the hot-spot, and hence the resistance.

Several types of superconducting detector are in widespread use for $\mathrm{mmw}$ and $\mathrm{THz}$ astronomy; their properties are described in an excellent recent review by Zmuidzinas and Richards[4]. The present detectors have many similarities to these, particularly to HEB mixers and transition-edge bolometers. Like them, our sensors are thermal, and strongly influenced by electrothermal feedback (ETF), a consequence of the narrow fractional width of the superconducting transition, i.e.

$$
\frac{T_{C}}{\Delta T}=\frac{T_{C}}{R} \frac{d R}{d T}>>1
$$

The effects of ETF were fully worked out, originally in the context of low-temperature semiconducting sensors, in classic papers by Mather[5,6] and Jones[7]. These treatments, along with the original treatments of HEB mixers[8] and TES bolometers[9], all treat the microbolometer as lumped, i.e. sufficiently small that temperature gradients within it may be neglected. On the other hand, large internal temperature gradients are essential to the operation of a hot-spot microbolometer. The theoretical treatment of their operation therefore closely parallels 1D treatments of HEB mixers[10], and is based on a simple 1D heat flow equation. Indeed, the governing equation for an HEB is identical to the hot-spot microbolometer's, with the addition of a term to represent the heat flow between the electrons and phonons in the film. In any case, solution of this $1 \mathrm{D}$ heat equation yields an I-V characteristic given by

$$
I=\frac{V}{R_{n}}\left\{1+2\left[\left(p_{0}+p_{e}-1\right)+\sqrt{\left(p_{0}+p_{e}+1\right)^{2}-4 p_{0}}\right]^{-1}\right\}
$$

where $p_{0}$ and $p_{e}$ are the (absorbed) $\mathrm{THz}$ and $\mathrm{DC}$ bias powers, normalized to an empirical "saturation power" $P_{\text {sat }}=4 \kappa w t\left(T_{c}-T_{0}\right) / l . \quad R_{n}=\frac{\rho l}{w t} \quad$ is the normal state resistance. This I-V curve reduces to $I=\frac{P_{S a t}}{V}+\frac{V}{R_{n}}$ in the small signal, low-background $\left(p_{0} \rightarrow 0\right)$ limit. Like HEB mixers (and unlike TES's), hot-spot microbolometers are wellmatched to lithographic antennas, with $R_{n}$ values (that determine the $\mathrm{THz}$ impedance-match) near $100 \mathrm{ohm}$. Unlike HEBs, their speed is governed by ordinary thermal (phonon) conduction along the bridge, not electron-phonon

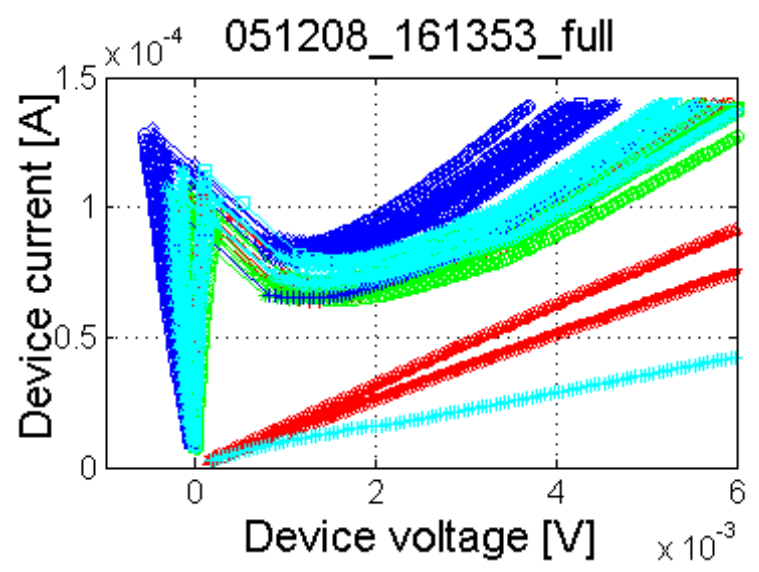

Fig. 3 I-V characteristics on a 32-element array. 
coupling, limiting signal bandwidths to $100 \mathrm{kHz}-1 \mathrm{MHz}$, depending on bridge dimensions.

Measured I-V curves fit theory quite well, and as shown in Fig. 3, uniformity is reasonable, particularly considering that fabrication of these devices is done in a commercial foundry. Performance is dominated by the three parameters $R_{n}, P_{s a t}$, and $T_{c}$. Responsivity for example, is given by $\left(4 R_{n} P_{\text {sat }}\right)^{-1 / 2}$.

Optical coupling to each bolometer is through a selfsimilar spiral antenna, with a hyperhemispherical Si lens mounted against the back side of the die, one lens centered behind the feedpoint of each of the 8 antennas. This arrangement[11] ideally produces a beam that (a) is circularlypolarized, (b) has flat frequency response over a wide fractional bandwidth given by the ratio of inner and outer radii of the spiral, and (c) has a width varying inversely with frequency, given by a uniform diffraction-limited aperture with the same diameter as the lens. The frequency response and beamwidth have been measured using the (less sensitive) room-temperature response of the microbolometers. The former is shown in Fig. 3, the latter is described in[12] and can be summarized as $F W H M=\frac{9 \mathrm{THz}-\operatorname{deg} r e e}{f}$.

\section{NIST bolometer frequency response}

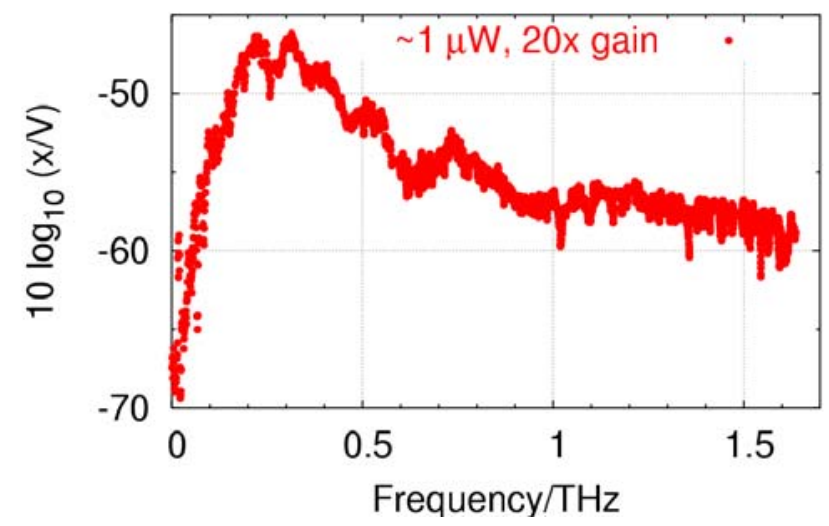

Fig.4. Frequency response of the uncooled detector, measured with a $\mathrm{CW}$ photomixer source (courtesy E. Brown.)

An important contrast with TES and HEB detectors is that the hot-spot microbolometer can be read out with appropriate room-temperature electronics without any degradation in noise performance. The novel low-noise readout scheme is described in[13] and illustrated in Fig.5. When the hot-spot microbolometer is biased at the minimum in its I-V curve, the dynamic impedance is extremely high. At that point a roomtemperature transimpedance pre-amplifier, with high enough noise impedance will not add significantly to the detector's Johnson and phonon noise.

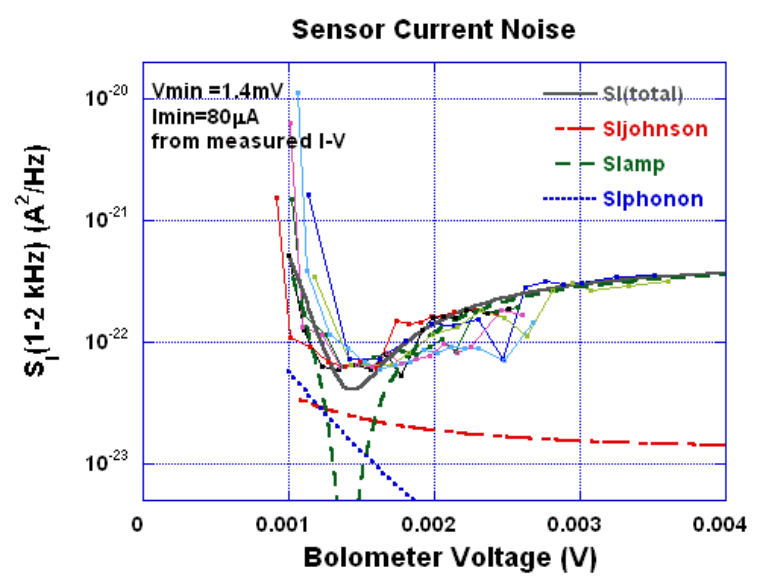

Fig. 5. Current noise vs. bias, illustrating the null in amplifier contribution at the point $(\sim 1.5 \mathrm{mV})$ where detector dynamic impedance becomes infinite.

\section{CAMERA ARCHITECTURE}

The camera's field-of-view is $2 \mathrm{~m}$ (high) $\mathrm{x} 4 \mathrm{~m}$ (wide) at a range of $8 \mathrm{~m}$ (i.e. $14 \times 28$ degrees). To attain a reasonable spatial resolution over this FOV, approx. 20 kipxels are needed. This is considerably larger than the most sophisticated $\mathrm{THz}$ arrays yet built, so a scanning architecture is mandatory. Our system uses a conical scanner near the aperture plane and a linear sensor array in the focal plane, as illustrated in Fig. 6.

Cryogenic cooling is provided by a commercial, closed-cycle, pulse-tube refrigerator, with a rated capacity of $250 \mathrm{~mW}$ at $4 \mathrm{~K}$. Although the refrigerator's power consumption is sizeable, at $\mathrm{xx} \mathrm{W}$, and forms the system's largest single power need, the refrigerator is surprisingly transparent to an enduser. The compressor is air-cooled, requiring only a standard single-phase $208 \mathrm{~V}$ outlet. The cooldown time is 15 hours.

To keep the system simple, and therefore robust and reliable, we use a "brute-force" (non-multiplexed) approach to array wiring. 300 wires are run from room-temperature to $4 \mathrm{~K}$, more than adequate for a 64-element array (4 wires/detector). An important practical issue is avoiding excessive heat load onto the $4 \mathrm{~K}$ stage from the wiring. This depends on the thermal conductivity vs temperature profile of the material. The wiring is implemented in 50-wire single-sided flex $(\mathrm{Cu}$ on kapton) cables with 100 um linewidth and 9 um thickness. We measure a heat load of $110 \mathrm{uW}$ per wire to the $4 \mathrm{~K}$ stage, using only $\sim 15 \%$ of the total cooling power. 


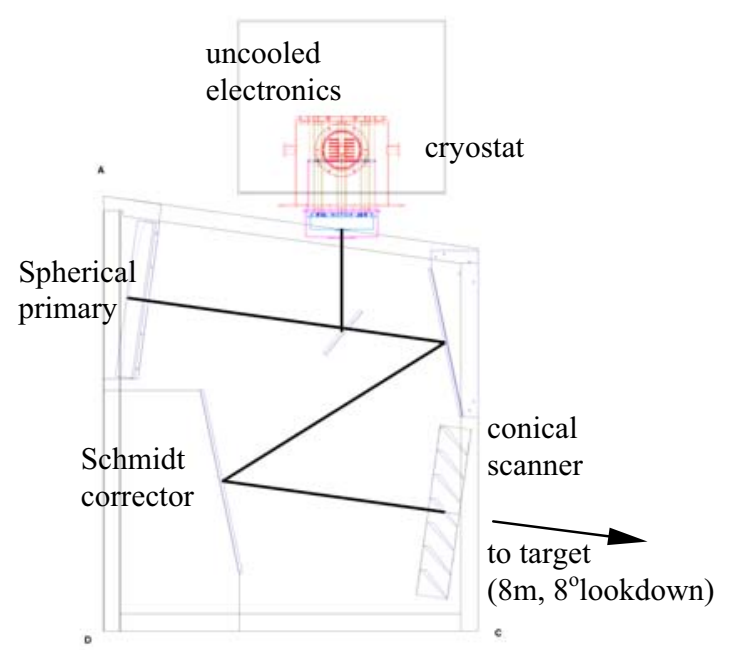

Fig. 6 Optical layout of the camera, with the downlooking cryostat shown at top. The compressor for the cryocooler and the control/acquisition computer are not shown.

\section{IMAGING}

A representative image taken with a single 8-element module and raster-scanned spherical primary is shown in Fig. 7. Significant "striping" from the non-uniform detector responsivity is visible, but can be removed with simple image processing. Further images are in the final presentation.

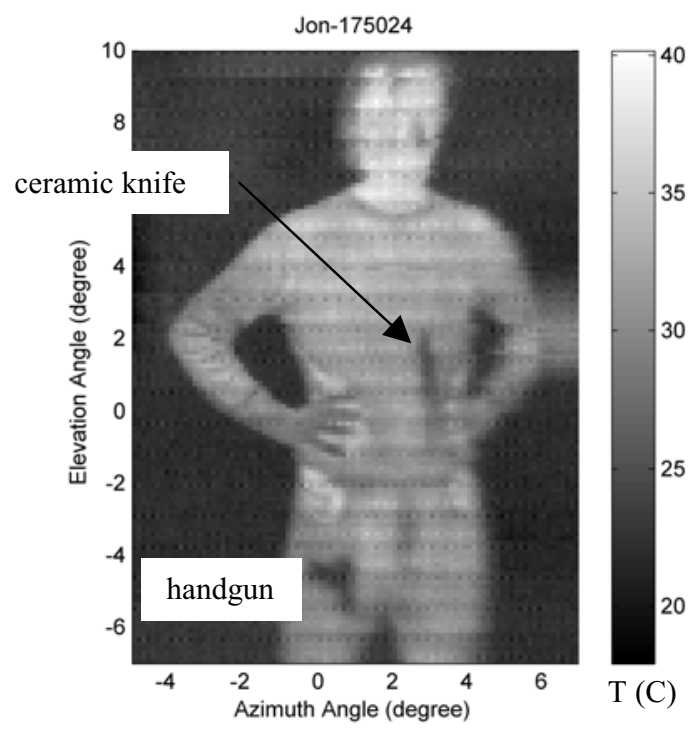

Fig. 7. Unprocessed passive image from a single module.

\section{Conclusion}

We have described the design and performance of a broadband passive terahertz camera, based on a linear array of superconducting hot-spot microbolometers. Single-pixel NETD is sufficiently low for these sensors to enable useful indoor personnel screening with a scanned system.

\section{ACKNOWLEDGEMENT}

The authors are grateful to the S\&T Directorate of DHS for sponsoring this work.

\section{REFERENCES}

[1] R. Appleby, and Wallace, H.B., "Standoff Detection of Weapons and Contraband in the $100 \mathrm{GHz}$ to $1 \mathrm{THz}$ Region," IEEE J. Anntennas and Propagation, vol. 55, pp. 2944-2956, 2007.

[2] A. Luukanen, and Pekola, J.P., "A Superconducting Antenna-coupled Hot-spot Microbolometer," Appl. Phys. Lett., vol. 82, pp. 3970-3972, 2003.

[3] A. Luukanen, Erich N. Grossman, Aaron J. Miller, Panu Helist"o, Jari S. Penttil"a, Hannu Sipola, and Heikki Sepp"a, "An Ultra-Low Noise Superconducting AntennaCoupled Microbolometer With a Room-temperature Readout," IEEE Microwave and Wireless Components Letters vol. 16, pp. 464-466, 2006.

[4] J. Zmuidzinas, and Richards, P.L., "Superconducting Detectors and Mixers for Millimeter and Submillimeter Astrophysics," Proc. IEEE, vol. 92, pp. 1597-1616, 2004. J. C. Mather, "Bolometer Noise: Nonequilibrium Theory," Appl. Optics, vol. 21, pp. 1125-1129, 1982.

[6] J. C. Mather, "Bolometers: ultimate sensitivity, optimization, and amplifier coupling," Appl. Optics, vol. 23, pp. 584-588, 1984.

[7] R. C. Jones, "The General Theory of Bolometer Performance," J. Optical Soc. of America, vol. 43, pp. 114, 1953.

H. Ekstrom, Karasik, B.S., Kollberg, E.L., Yngvesson, K.S., "Conversion Gain and Noise of Niobium Superconducting Hot-Electron Mixers," IEEE Trans. Microwave Theory and Tech., vol. 43, pp. 938-947, 1995.

A. T. Lee, Richards, P.L., Nam, S.W., Cabrera, B., and Irwin, K.D., "A superconducting bolometer with strong electrothermal feedback," Appl. Phys. Lett., vol. 69, pp. 1801-1803, 1996.

H. Merkel, Khosropanah, P., Floet, D.W., Yagoubov, P.A., and Kollberk, E.L., "Conversion Gain and Fluctuation Noise of Phonon-Cooled Hot-Electron Bolometers in HotSpot Regime," IEEE Trans. Microwave Theory and Tech.. vol. 48, pp. 690-699, 2000.

T. H. Buettgenbach, "An Improved Solution for Integrated Array Optics in Quasi-optical Mm and Submm Receivers," IEEE Trans. Microwave Theory and Tech. vol. 41, pp. 1750-1761, 1993.

C. Dietlein, Chisum, J.D., Ramirez, M.D., Luukanen, A., Grossman, E.N., Popovic, Z., "Integrated Microbolometer Antenna Characterization from $95-650 \mathrm{GHz}$," Intl. Microwave Symposium Digest, pp. 1165-1168, 2007.

[13] J. S. Penttila, Sipola, H., Helisto, P., and Seppa, H., "Lownoise Readout of Superconducting Bolometers based on Electrothermal Feedback," Superconductor Science and Technology, vol. 19, pp. 319-322, 2006. 